\title{
Consumer health awareness booklet: a guide to intelligent decisions for selecting integrative holistic medicine: adding complementary and alternative medicine to science based healthcare
}

\section{Integrative and holistic medicine}

Integrative medicine is a revolutionary approach emerging to represent a broader healing paradigm of medicine than the biomedical model alone. ${ }^{1}$ Integrative medicine is often referred to as wholistic health care and or holistic medicine. "Scientific practitioners regard holistic medicine as treatment of the "whole patient," with due attention to emotional factors as well as the patient's lifestyle". ${ }^{2}$ This method of medicine is patient centered and healing oriented, integrates conventional medicine and naturopathic medicine, alternative and complementary therapies, and involves a paradigm shift from illness to health (Figure 1). ${ }^{3}$

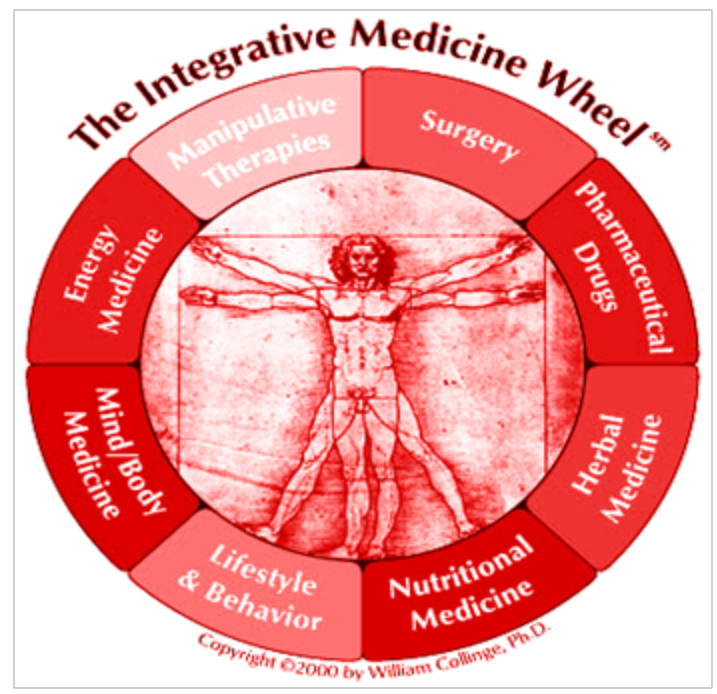

Figure I The Integrative Medicine Wheel Combines Traditional Medicine and Complementary and Alternative Medicine Treatment Modalities.

\section{Integrative and holistic medicine attributes}

a. Integrative and holistic medicine is used by both scientific and nonscientific health care practitioners.

b. The therapeutic approach of integrative and holistic medicine includes preventive health care, therefore requiring less sick care.

c. The holistic approach is an important modern development in the health-care field that is commonly associated with naturopathic care.

d. Practitioners of integrative medicine synthesize standard and alternative methods, using the best of both (Figure 2)., ${ }^{2,3}$

\section{Complementary and alternative medicine}

The National Institutes of Health Center for Complementary and Alternative Medicine (NCCAM) define and describe the "CAM"

\author{
Volume II Issue 3 - 2018
}

\author{
Robelyn A Garcia ${ }^{1,2}$ \\ 'Arizona State University, USA \\ ${ }^{2}$ Harvard University, USA
}

Correspondence: Robelyn A Garcia, Arizona State University, USA, Email Dr.RobelynGarcia@asu.edu, RoG8I4@g.harvard. edu

Received: April 19, 2018 | Published: May 09, 2018

movement as a group of diverse medical and health care systems, practices, and products that are not yet considered to be part of traditional medicine. ${ }^{2}$ Complementary medicine is used in conjunction with conventional medicine, and alternative medicine is sometimes used in place of traditional western medicine. CAM treatments are often used in addition to and integrated with traditional medical treatments.

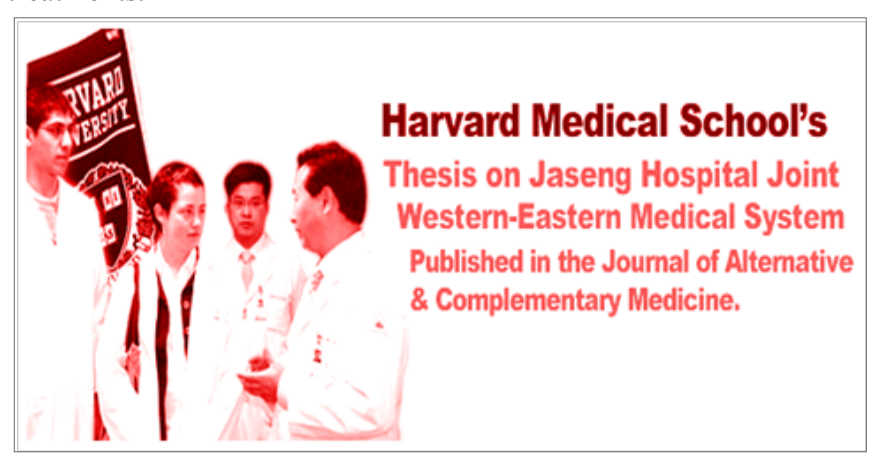

Figure 2 Harvard Medical School's Thesis Supporting Integrative Medicine at Jaseng Hospital - Published in The Journal of Alternative and Complementary Medicine.

\section{Complementary and alternative medicine practices (Figure 3)}

\section{Complementary and alternative medicine domains}

The NCCAM classifies CAM therapies into the five illustrated domains:
a. Whole Medical Systems.
b. Mind-Body Medicine.
c. Biologically Based Practices.
d. Manipulative and Body-Based Practices.
e. Energy Medicine ${ }^{2}$ 


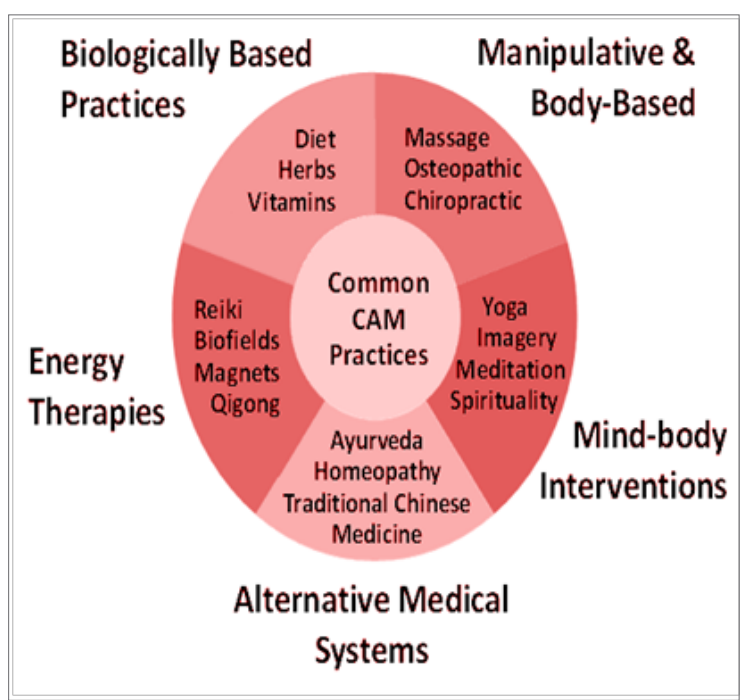

Figure 3 Complementary and Alternative Medicine Treatment Domains from Harvard Presentation "Alternative to What? Complementary to Whom?"

\section{Complementary and Alternative Medicine Methods}

"A complete listing of "CAM" methods would be a monumental task". ${ }^{2}$ Some of the most recognized CAM therapeutic approaches include but are not limited to mind-body medicine (Yoga Therapy, Tai Chi, Pilates, Meditation), acupuncture, quantum healing, massage therapy, animal-assisted therapy, nutritional supplements, detoxification, biofeedback, neuro feedback, evidence based selfcare, chiropractic care, vitalism, Chinese medicine, homeopathy, naturopathy, spiritual healing, ritual healing, clinical and applied hypnosis, guided imagery, herbal medicines, aromatherapy, touch therapy, energy therapy, art therapy, music therapy, and rhythmic auditory stimulation (Figure 4). ${ }^{2,4}$
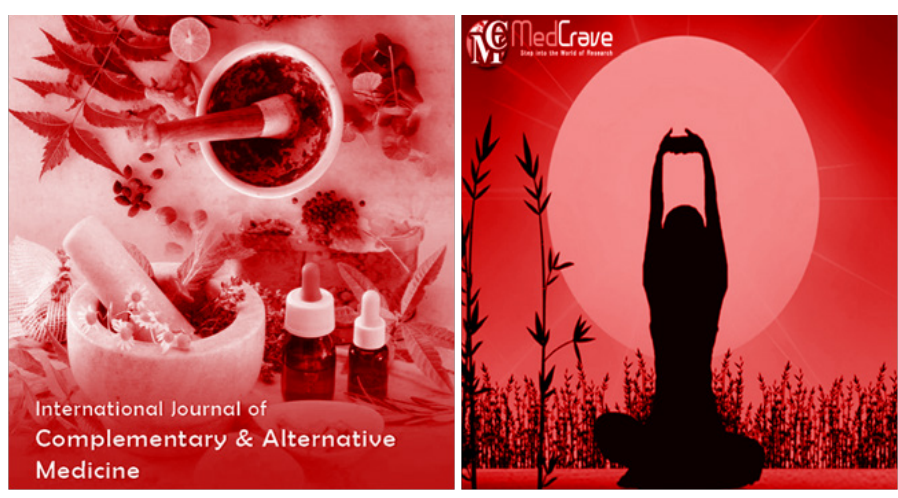

Figure 4 International Journal of Complementary and Alternative Medicine Peer Reviewed Open Access Articles and Research Available to Consumers.

\section{Complementary and alternative medicine open access}

A good doctor treats the symptoms of a disease; the best doctor treats the patient who has a disease with all available and effective therapies.

\section{Thoughts on choosing an integrative holistic doctor}

a. The best time to look for a doctor or clinic is before you become ill. b. Improved health outcomes stem from a mutual partnership between the patient and the doctor.

c. Health care treatment that is good for one patient may not be what another patient needs or prefers.

d. The best protection against unnecessary surgery is a competent doctor.

e. Employ selection of health care and wellness practitioners with great thought and care.

f. Be cautious of treatments that lack empirical research data, scientific support and rationale.

g. Consumer knowledge is of import in making informed health decisions. ${ }^{2,5}$

\section{Top ten qualities of an integrative holistic doctor}

a. Intelligent, knowledgeable, educated, licensed and certified.

b. Sympathetic and interested in the patient.

c. Provides traditional, preventive, complementary \& alternative therapy.

d. Keeps up-to-date by reading journals and attending conferences.

e. Provides clear explanation of the diagnosis and treatment to patient.

f. Completes annual Continuing Medical Education (CME).

g. Treats the patient as a whole being, mind and body as one.

h. Serves as the patient's support and healer.

i. Publishes peer reviewed articles or reviews another doctors' research.

j. Is conservative about recommending surgery. ${ }^{2,3}$

\section{Acknowledgments}

None

\section{Conflict of interest}

Author declares there is no conflict of interest.

\section{References}

1. Garcia R. Integrative Mind-Body Medicine as a Treatment for Psychophysiologic Disorders Utilizing the Seven Keys to Treating Stress Illness. Int J Complement Alt Med. 2015;1(3):00017.

2. Barrett S, London WM, Kroger M, et al. Consumer health: A guide to intelligent decisions. 9th ed. New York: McGraw-Hill Education; 2003.

3. http://www.drshawntassone.com/services/integrative-medicine

4. Garcia R, Benavidez D. Transtheoretical Model Key Constructs Applied to the Intervention \& Treatment of Weight Cycling \& Yoyo Dieting Cognitive-Affective Bases of Health for Weight Management. Int $J$ Complement Alt Med. 2016;3(3):00071.

5. https://integrativemedicine.arizona.edu/about/definition.html 